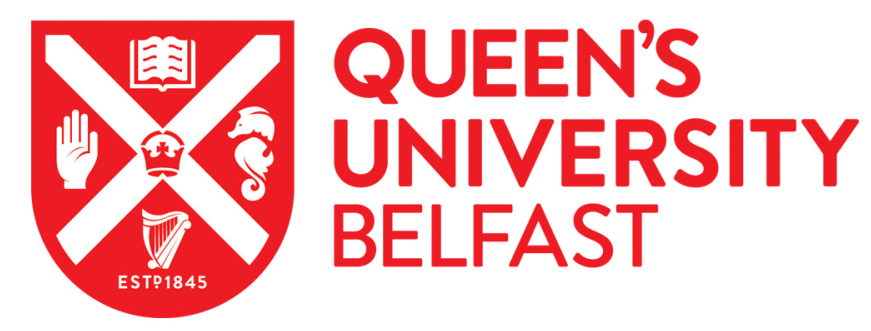

\title{
The effect of positively chirped laser pulse on energy enhancement of proton acceleration in combinational radiation pressure and bubble regime
}

Vosoughian, H., Sarri, G., Borghesi, M., Hajiesmaeilbaigi, F., \& Afarideh, H. (2017). The effect of positively chirped laser pulse on energy enhancement of proton acceleration in combinational radiation pressure and bubble regime. Physics of Plasmas, 24(10), [103123]. https://doi.org/10.1063/1.4999385

Published in:

Physics of Plasmas

Document Version:

Publisher's PDF, also known as Version of record

Queen's University Belfast - Research Portal:

Link to publication record in Queen's University Belfast Research Portal

Publisher rights

(c) 2017 AIP Publishing. This work is made available online in accordance with the publisher's policies. Please refer to any applicable terms of use of the publisher

\section{General rights}

Copyright for the publications made accessible via the Queen's University Belfast Research Portal is retained by the author(s) and / or other copyright owners and it is a condition of accessing these publications that users recognise and abide by the legal requirements associated with these rights.

\section{Take down policy}

The Research Portal is Queen's institutional repository that provides access to Queen's research output. Every effort has been made to ensure that content in the Research Portal does not infringe any person's rights, or applicable UK laws. If you discover content in the Research Portal that you believe breaches copyright or violates any law, please contact openaccess@qub.ac.uk. 
The effect of positively chirped laser pulse on energy enhancement of proton acceleration in combinational radiation pressure and bubble regime

H. Vosoughian, G. Sarri, M. Borghesi, F. Hajiesmaeilbaigi, and H. Afarideh

Citation: Physics of Plasmas 24, 103123 (2017); doi: 10.1063/1.4999385

View online: http://dx.doi.org/10.1063/1.4999385

View Table of Contents: http://aip.scitation.org/toc/php/24/10

Published by the American Institute of Physics

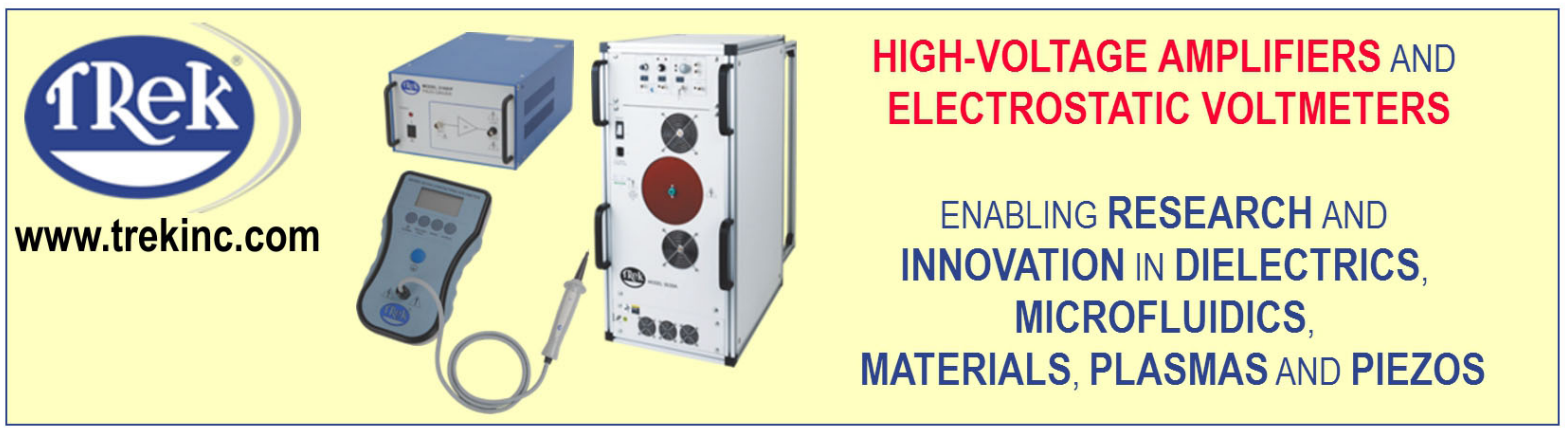




\title{
The effect of positively chirped laser pulse on energy enhancement of proton acceleration in combinational radiation pressure and bubble regime
}

\author{
H. Vosoughian, ${ }^{1,2,3, a)}$ G. Sarri, ${ }^{2}$ M. Borghesi, ${ }^{2}$ F. Hajiesmaeilbaigi, ${ }^{1}$ and H. Afarideh ${ }^{3}$ \\ ${ }^{1}$ Photonics and Quantum Technologies Research School, Nuclear Science and Technology Research Institute, \\ Tehran, Iran \\ ${ }^{2}$ School of Mathematics and Physics, the Queen's University of Belfast, BT7 INN Belfast, United Kingdom \\ ${ }^{3}$ Department of Energy Engineering and Physics, Amirkabir University of Technology, Tehran, Iran
}

(Received 8 August 2017; accepted 26 September 2017; published online 10 October 2017)

Proton energy enhancement in a combinational radiation pressure and bubble regime by applying a positively chirped laser pulse has been studied using a series of two-dimensional particle-in-cell simulations. In this regime, the proton injection in the half-first period of an excited plasma wave in an under-dense plasma plays the main role in the acceleration process. Moreover, exciting as high as large-amplitude plasma waves can significantly increase the conversion efficiency of laser energy into kinetic energy of the trapped protons. Here, the utilization of the positively chirped laser pulse is proposed as an effective approach to excite the higher amplitude wake in the combinational regime. Our studies indicate that in the positively chirped combinational regime, the plasma wake with approximately two-fold enhancement is produced that results in the generation of the proton bunch with the narrower energy spread and also the peak enhancement by a factor of two, compared with the un-chirped one. This improvement in proton energy reveals that the chirped laser pulse can be introduced as a tool to tune the energy of generated protons in the combinational radiation pressure and bubble regime. Published by AIP Publishing.

https://doi.org/10.1063/1.4999385

\section{INTRODUCTION}

Recently, laser-plasma accelerators as an alternative to the conventional particle accelerators, have attracted more attention due to the huge acceleration rate, and thus they are potential for making compact accelerators. ${ }^{1-3}$ In the last decade, different mechanisms have been proposed to accelerate the charged particles via laser-plasma interactions. ${ }^{4-10}$ Depending on the species of particles (electrons or ions), a static electric field (ion acceleration) or a running electric wave (electron acceleration) is used to accelerate the particles. The electron acceleration mechanism is based on the Wakefield regime in which the ponderomotive force of the laser pulse excites a plasma wave when the laser pulse propagates through the under-dense plasma. Under proper conditions, the ambient plasma electrons are trapped inside the electric field of the excited wave and accelerated to the relativistic energies. ${ }^{11}$ In the well-known ion acceleration regimes such as the Target Normal Sheath Acceleration (TNSA), ${ }^{12,13}$ the Radiation Pressure Acceleration (RPA), ${ }^{14-16}$ and the Coulomb Explosion, ${ }^{17-19}$ an induced electrostatic field within the over-dense target, plays the main role in the acceleration process. Among the mentioned mechanisms, the RPA is promising approach to achieve the ion beams with ion energy in the range of $\mathrm{GeV} .{ }^{14}$ Based on this technique, due to pulse reflection from a dense plasma which has the density several times the critical density, the ponderomotive force of the laser pulse pushes the electrons forward leaving behind a positive ion space charge. When the target is sufficiently thin, the rear surface of the target

\footnotetext{
a)vosough_phn@yahoo.com
}

(and sometimes entire target) begins to move, while the laser interacts with the target. When the target is pushed with the laser ponderomotive force (such as the circularly polarized laser pulse) without too much heating of electrons, ions in the target as a whole are trapped in an accelerating bucket with tight phase space circles and gain more energy and enter into the relativistic domain. ${ }^{20}$ However, the presence of some instabilities such as Rayleigh-Taylor ${ }^{21}$ and Weibel ${ }^{22,23}$ limit the maximum attainable ion energy during the RPA. To overcome these limitations, combinational regimes have been proposed..$^{24-27}$ As shown theoretically, it is possible to generate the ion beams with ion energy in excess of tens and hundreds of $\mathrm{GeV}$ by combination of the RPA and the bubble regime driven by a single Super-Gaussian (SG) laser pulse in the under-dense plasma. ${ }^{28-31}$ The main idea in this method is injection of pre-accelerated ions inside the half-first period of the excited plasma wave in the under-dense plasma. By this way, the protons in the dense plasma, adhered to the under-dense plasma, are firstly pre-accelerated by the radiation pressure of an ultra-short Circular Polarized SG laser pulse. Once the laser pulse passes through the dense target and enters the under-dense plasma, the protons are trapped in the bubble structure driven by the SG laser pulse and stably re-accelerated. The two layered solid target, ${ }^{32}$ an underdense plasma with a gradient density (to control phase velocity of Wakefield), ${ }^{33,34}$ and applying an ultra-intense Laguerre-Gaussian (LG) laser pulse, ${ }^{35}$ can increase the efficiency of energy transferred to the injected protons in this regime. In this paper, a novel sequential acceleration scheme based on applying a positively chirped laser pulse has been proposed to improve the effectiveness of the acceleration 

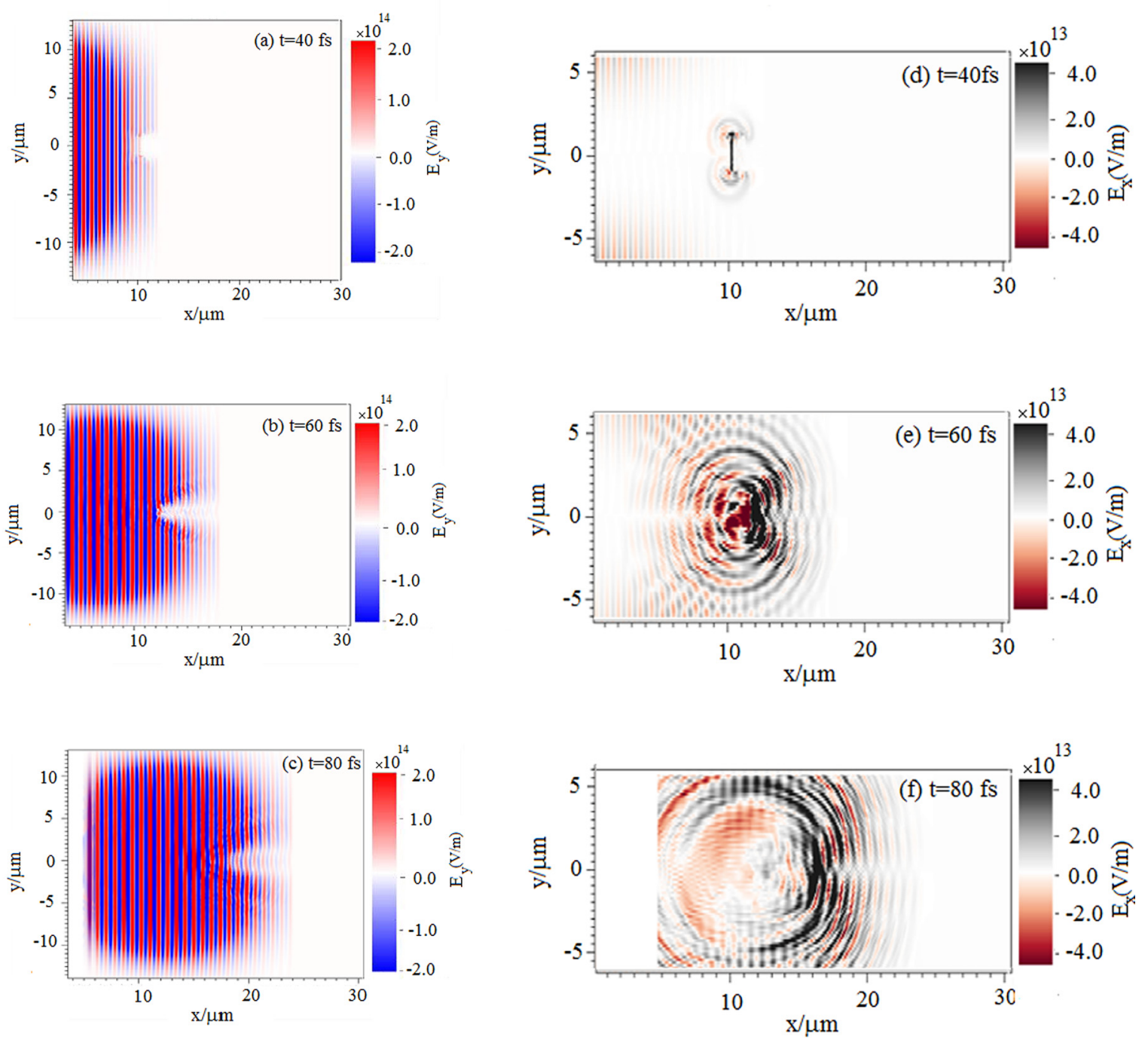

FIG. 1. The 2D spatial distribution of the electric fields during the pure RPA regime from PIC simulations. (a)-(c): The transverse profile of the laser's electric field, $E_{y}$, interacting with the single micro-target, (d-f): The transverse profile of the longitudinal electrostatic field within the solid target, $E_{x}$, at $\mathrm{t}=40 \mathrm{fs}, \mathrm{t}=60 \mathrm{fs}$, and $\mathrm{t}=80 \mathrm{fs}$.

process. Based on this technique, a considerable difference in amplitude of the excited plasma wave in the under-dense plasma is observed for the positively chirped laser pulse than the un-chirped one. ${ }^{36-38}$ This difference leads to increase of the energy of trapped ions in the wake, and hence, improvement of the quality of the injected bunch in the combinational radiation pressure and bubble regime. The simulation results show that protons of $14 \mathrm{GeV}$ are obtained in the combinational regime when using the SG positively Chirped Laser pulse with an intensity of $I=2.14 \times 10^{23} \mathrm{Wcm}^{-2}$. Compared with the Sequential Un-chirped laser pulse acceleration scheme, the combinational chirped Laser pulse scheme can accelerate protons to higher energy without using higher laser intensity.

\section{SIMULATION MODE}

In order to demonstrate the proposed scheme, a series of two-dimensional particle-in-cell (2D PIC) simulations have been carried out by the EPOCH code ${ }^{39}$ to show the effect of pulse chirping on the evolutions of Wakefield amplitude. In these simulations, the Circularly Polarized (CP) laser pulse is temporally Gaussian and transversely fourth-order SuperGaussian (SG) that is frequency chirped as ${ }^{37}$

$$
\begin{aligned}
\vec{E}_{L}(y, \xi)= & E_{0} \exp \left(-y^{4} / \sigma_{y}^{4}\right) \exp \left(-\xi^{2} / \sigma_{x}^{2}\right) \\
& \times\left[\cos (\omega(\xi) \xi) \hat{e}_{y}+\sin (\omega(\xi) \xi) \hat{e}_{z}\right] .
\end{aligned}
$$

Here, the electric field amplitude of the laser pulse is defined as $E_{0}$, which is a constant value. The local frequency of the chirped pulse, the longitudinal pulse length, and the laser beam waist is denoted by $\omega(\xi)=(1-b \xi), \sigma_{x}$, and $\sigma_{y}$, respectively. Here, $b>0(b<0)$ indicates the dimensionless chirp parameter for the positively (negatively) chirped pulse and $\xi=\frac{(x-c t) \omega_{0}}{c}$ is the dimensionless retarded time in which $c, \omega_{0}, x$, and $t$ are the speed of light in vacuum, the local frequency in the center of the pulse, the spatial coordinate in the propagation axis direction, and the temporal coordinate, respectively. In this work the normalized laser amplitude of $a_{0}=\frac{e E_{0}}{m_{c} c \omega_{0}}=316$ is taken, which corresponds to the laser intensity of $I=2.14 \times 10^{23} \mathrm{Wcm}^{-2}$. The laser beam waist and the longitudinal pulse length are also set equal to $\sigma_{y}=$ $16 \mu \mathrm{m}$ and $\sigma_{x}=10 \mu \mathrm{m}$, respectively. The simulation box is $64 \mu \mathrm{m}(x) \times 150 \mu \mathrm{m}(y)$ that corresponds to a moving window 
(a)

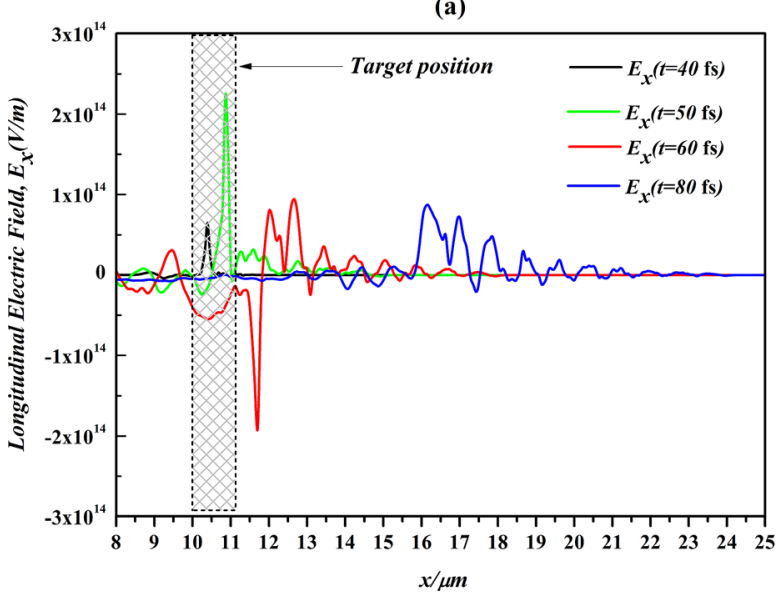

(b)

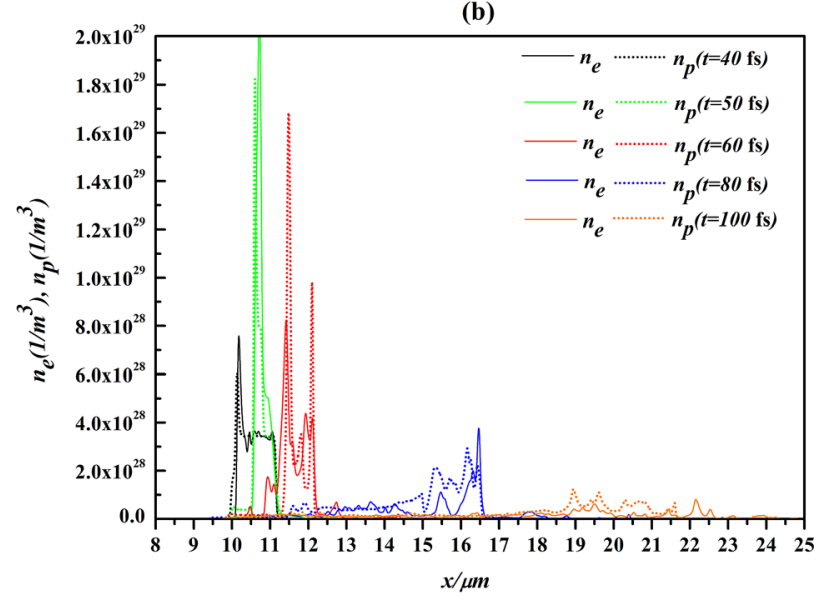

FIG. 2. The 1D spatial distribution of: (a) the longitudinal electrostatic field and (b) the density of protons within the solid target at $t=40 \mathrm{fs}-100 \mathrm{fs}$. As is clear, the electrostatic field at the rear side of the target is turned into the dipole-like structure during the time and therefore caused to spread across the proton layer therefore degrading the RPA mechanism.

with $2000 \times 900$ cells and there are 4 particles per cell. The left vacuum is $10 \mu \mathrm{m}$ and the proton-rich micro-target with the density of $20 n_{c}$ is located in the region of $10 \mu \mathrm{m}<x<$ $11.2 \mu \mathrm{m}$ and $-1.2 \mu \mathrm{m}<y<1.2 \mu \mathrm{m}$. The laser pulse enters the simulation box from the left boundary. The under-dense helium plasma $\left(T_{e}=0\right)$ is initially located in the region of $11.2 \mu \mathrm{m}<x<1600 \mu \mathrm{m}$ and $-75 \mu \mathrm{m}<y<75 \mu \mathrm{m}$ with the density of $n_{e}=0.02 n_{c}$. Here, $n_{c}=1.1 \times 10^{21} \mathrm{~cm}^{-3}$ is the critical density for the laser pulse with the wave length of $\lambda_{L}=1 \mu \mathrm{m}$.

\section{RESULTS AND DISCUSSION}

To show the effectiveness of the sequential acceleration scheme, the properties of the generated proton bunch from a dense micro-target during the pure RPA mechanism have been considered, first. In this regards, the 2D spatial distribution of the laser's electric field, $E_{y}$, and the longitudinal electrostatic field, $E_{x}$, during the time are presented in Figs. 1(a)-1(c) and Figs. 1(d)-1(f), respectively. As it is obvious from these figures, the transverse size of the micro-target is much smaller than the laser focal spot and thus, the major part of the abaxial laser energy propagates along the edges to the rear side of the target [Figs. 1(b) and 1(c)], whereas the axial portion of the laser experiences much heavier reflection.

Accordingly, due to the pressure of the reflected light, the plasma electrons are compressed to a highly dense electron layer in the forward direction that leads to establishment of the longitudinal electrostatic field within the solid target [Fig. 1(d)]. However, due to wave front curvature at the target rear surface, the uniform spatial distribution of the electrostatic field turns to a spherical-like wave structure during the time [as seen in Figs. 1(e) and 1(f)]. Moreover, the 1-D spatial distribution of the electrostatic field along the propagation axis [Fig. 2(a)] reveals that the wave interface effect causes the electrostatic field to take a dipole-like spatial distribution over the acceleration area, which decompresses the electron layer consequently spreading the protons density during the time as shown in Fig. 2(b).
The energy spectrum of generated protons is presented in Fig. 3. As seen, the proton energy spectrum tends to the Quasi-Maxwellian distribution function during the time (the green line in the inset of Fig. 3). In fact, the presence of the dipole-like field causes the protons to experience the acceleration and deceleration modes, successively, which redistribute the energy between particles and make the velocity distribution isotropic.

However, by inserting the under-dense plasma behind the dense micro-target, the accelerated protons via the RPA mechanism can be injected into the electric field of the produced bubble when the transmitted laser pulse propagates through the under-dense plasma. The simulation results of the sequential acceleration scheme have been shown in Fig. 4. As it is obvious, the pre-accelerated protons (the brown line) can ride the plasma wake [Fig. 4(a)] staying in phase with the longitudinal electric field inside the wake (the red line) and gaining more energy than the pure RPA. In Fig. 4(b), the energy spectrum of the trapped electrons inside the Wakefield during the sequential acceleration regime at $t=610$ fs (purple curve) has

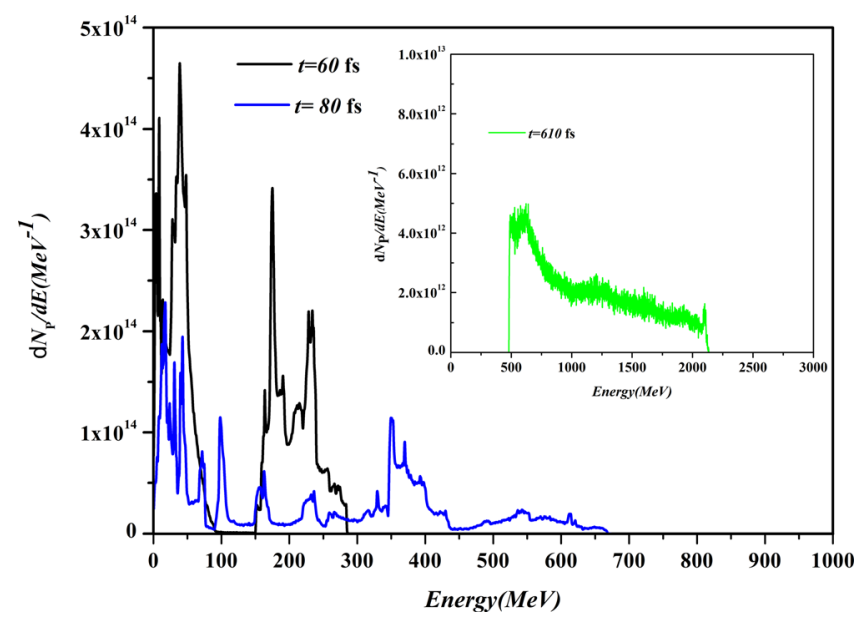

FIG. 3. The energy spectrum of generated protons when the laser is incident on the single dense micro-target at $t=60 \mathrm{fs}$ and $\mathrm{t}=80 \mathrm{fs}$. The inset shows the energy spectrum of accelerated protons at $t=610 \mathrm{fs}$ that indicates the spreading of the energy spectrum during the interaction time. 
(a)

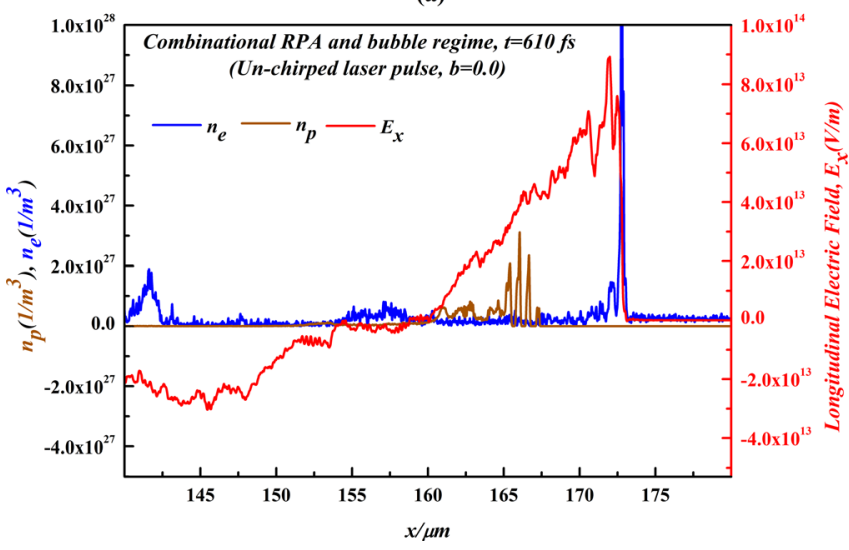

(b)

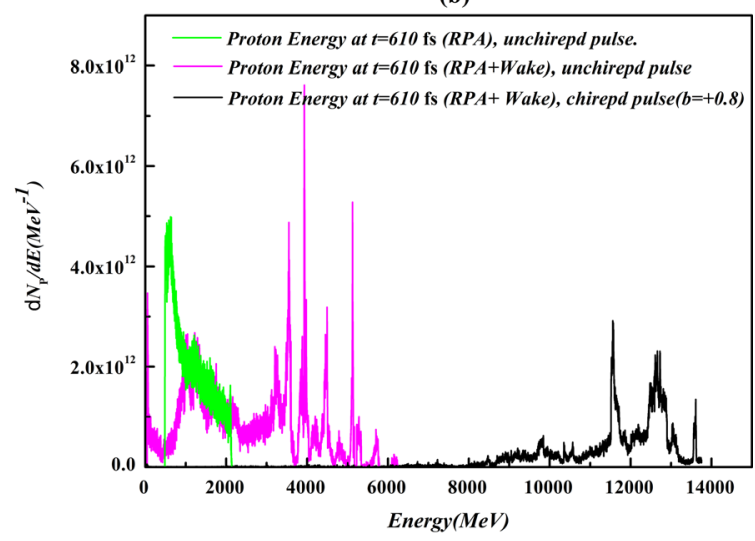

FIG. 4. The PIC simulation results of the sequential acceleration scheme. (a) Snapshots of the electron density (blue line), accelerating field of wake (red line), and injected protons in the wake (brown line) at $t=610 \mathrm{fs}$, where the un-chirped laser pulse is used in the combinational RPA and bubble regime. (b) The energy spectrum of generated protons from the pure RPA (green line) mechanism, the un-chirped combinational RPA, and the bubble regime (purple line), and the positively chirped combinational RPA and bubble regime (black line).

been compared with that of the pure RPA mechanism (the green line). It is found that the protons with maximum energy, about 3-fold higher than the pure RPA and also with lower energy spread, are produced during the acceleration process.

Motivated by the Wakefield electron acceleration, ${ }^{40}$ the energy of trapped protons, inside the bubble in the sequential acceleration regime, can be improved by exciting the higher amplitude plasma wake. As it is demonstrated, ${ }^{37}$ by locating the lower frequencies in front of the pulse, the plasma transparency decreased at the leading part of the pulse resulting in the excitation of a large density spike and also a stronger wake-field compared with the un-chirped laser pulse. The amplitude of the excited wake for different types of chirped laser pulse has been shown in Fig. 5(a). The outcomes reveal that the wake's amplitude is increased when the positively chirped laser pulse with higher chirped parameter, $b$, propagates through the under-dens plasma. In order to show the effectiveness of the positively chirped laser pulse on the enhancement of protons energy in the sequential regime, the acceleration process has been shown in Fig. 5(b) by applying the positively chirped pulse with the chirp parameter of $b=+0.8$. As it is evident, the Wakefield's amplitude takes the amount of $E_{x} \sim 1.4 \times 10^{14}(\mathrm{~V} / \mathrm{m})$ which is approximately 2 -fold more than its value when the un-chirped laser pulse is used $\left(E_{x} \sim 8 \times 10^{13}(\mathrm{~V} / \mathrm{m})\right)$. Therefore, it can be attributed to more effective energy exchange from the wake to the trapped protons. The energy of accelerated protons in the Positively Chirped Sequential (PCS) regime, at $t=610$ fs, is shown in Fig. 4(b) by the black line. It is seen that the proton bunch with the maximum energy of about $14 \mathrm{GeV}$ produced in the PCS regime shows significant enhancement in the acceleration process.

To show the quality of the produced bunch in more detail, two dimensional spatial distributions of the trapped protons in the excited wave are presented in Fig. 6(a) for the Un-Chirped Sequential (UCS) regime and in Fig. 6(b) for the Positively Chirped Sequential (PCS) regime. As is clear, though the transverse divergence of the beam is increased during the PCS regime, the beam's longitudinal emittance is greatly improved in the case of the PCS regime.

The reason is that when the un-chirped laser pulse enters into the under-dense plasma, the bubble structure with an electron pillar on its axis emerges, as shown in Fig. 7(a). The (a)

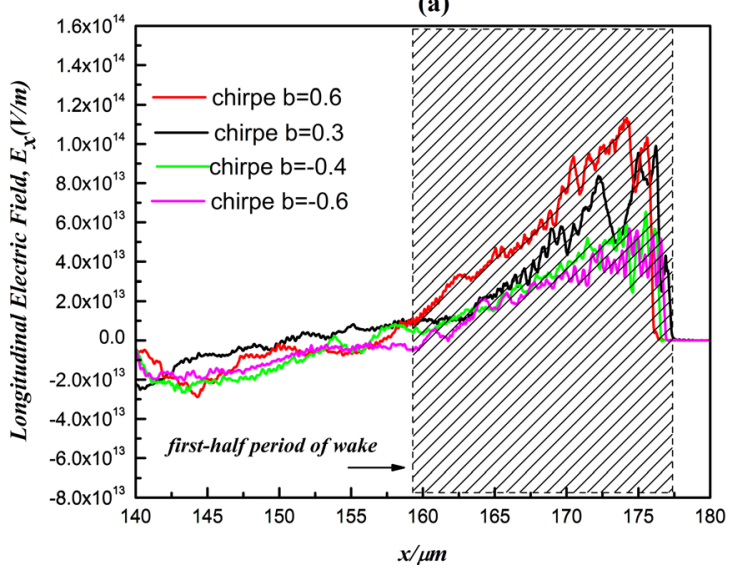

(b)

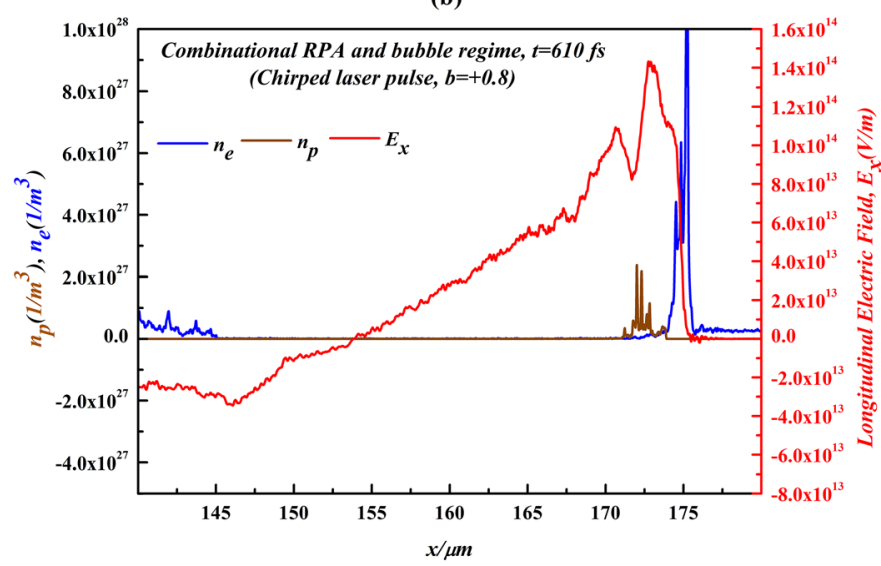

FIG. 5. The PIC simulation results of the positively chirped sequential acceleration scheme. (a) The amplitude of the excited wake for different types of chirped laser pulses when propagating in the under-dense plasma. (b) Snapshots of the electron density (blue line), accelerating field of wake (red line), and injected protons in the wake (brown line) at $\mathrm{t}=610 \mathrm{fs}$, where the positively chirped laser pulse of $b=+0.8$ is used. 

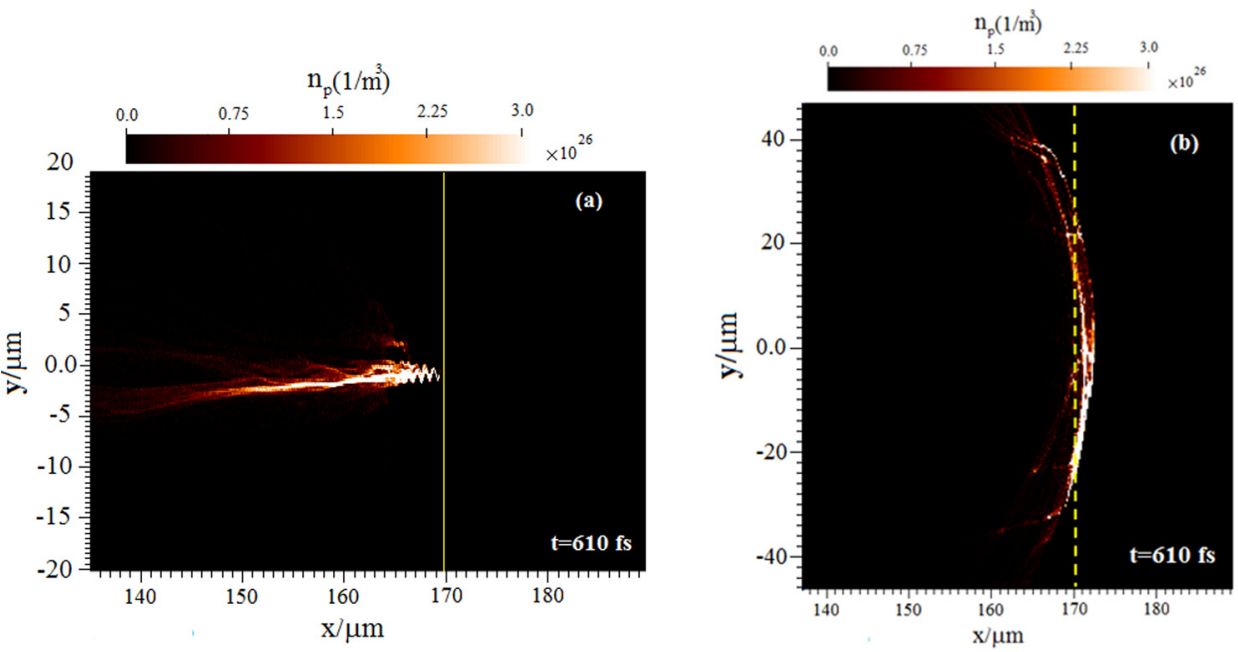

FIG. 6. The 2D spatial distribution of the trapped protons in the excited wave for: (a) the Un-Chirped Sequential (UCS) regime and (b) the Positively Chirped Sequential (PCS) regime.
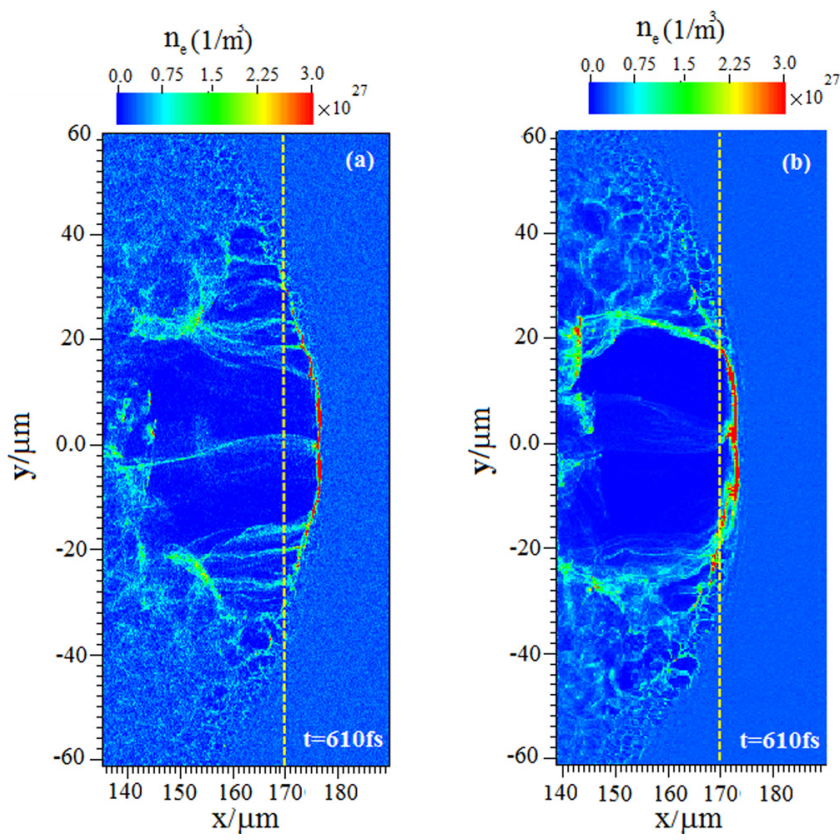

FIG. 7. The 2D spatial distribution of the plasma electrons in the cases of: (a) the Un-Chirped Sequential (UCS) regime and (b) the Positively Chirped Sequential (PCS) regime. As obvious, in the case of the UCS regime, an electron pillar appears on the propagation axis that plays an important role in producing a strong focusing field on the bubble structure.

electron pillar provides a strong focusing force, which is expressed as $F_{y} \propto\left(E_{y}-c B_{z}\right)$, near the $x$ axis in the transverse direction [as demonstrated in Ref. 31].Here, $E_{y}$ and $B_{z}$ are the electric and magnetic fields, respectively. Therefore, due to the absence of the electron pillar in the case of the positively chirped laser pulse [Fig. 7(b)], the focusing force changes to defocusing force and therefore growing a divergence of the accelerated bunch.

\section{CONCLUSIONS}

As the energy enhancement of accelerated protons in a combinational radiation pressure and bubble regime depends on the Wakefield amplitude, to produce higher energy particles in this regime, the plasma wake with the largest amplitude must be excited through the under-dense plasma. Since locating the lower frequencies in front of the pulse leads to decreasing the plasma transparency at the leading part of the pulse, therefore larger density spike and also a stronger wake-field are produced when the positively chirped pulse is used in the combinational regime. As a result, the efficiency of energy transferred to the injected protons is improved that leads to production of higher energy protons $(14 \mathrm{GeV})$ compared with the sequential scheme where the un-chirped laser pulse has been applied $(6 \mathrm{GeV})$. This difference in proton energy indicates that the positively chirped pulse could be introduced as an effective tool to control the quality of produced protons in a combinational radiation pressure and bubble regime.

${ }^{1}$ T. Tajima and J. M. Dawson, Phys. Rev. Lett. 43, 267 (1979).

${ }^{2}$ V. Malka, S. Fritzler, E. Lefebvre, M. M. Aleonard, F. Burgy, J. P. Chambaret, J. F. Chemin, K. Krushelnick, G. Malka, S. P. D. Mangles, Z. Najmudin, M. Pittman, J. P. Rousseau, J. N. Scheurer, B. Walton, and A. E. Dangor, Science 298, 1596-1600 (2002).

${ }^{3}$ S. V. Bulanov, M. Yamagiwa, T. Z. Esirkepov, D. V. Dylov, F. F. Kamenets, N. S. Knyazev, J. K. Koga, M. Kando, Y. Ueshima, K. Saito, and D. Wakabayashi, Plasma Phys. Rep. 32, 263 (2006).

${ }^{4}$ A. Pukhov and J. Meyer-ter-Vehn, Appl. Phys. B 74, 355 (2002).

${ }^{5}$ S. P. D. Mangles, C. D. Murphy, Z. Najmudin, A. G. R. Thomas, J. L. Collier, A. E. Dangor, E. J. Divall, P. S. Foster, J. G. Gallacher, C. J. Hooker, D. A. Jaroszynski, A. J. Langley, W. B. Mori, P. A. Norreys, F. S. Tsung, R. Viskup, B. R. Walton, and K. Krushelnick, Nature 431, 535 (2004).

${ }^{6}$ V. Malka, J. Faure, Y. Glinec, A. Pukhov, and J. P. Rousseau, Phys. Plasmas 12, 056702 (2005).

${ }^{7}$ W. P. Leemans, B. Nagler, A. J. Gonsalves, C. Tóth, K. Nakamura, C. G. R. Geddes, E. Esarey, C. B. Schroeder, and S. M. Hooker, Nat. Phys. 2 , 696 (2006)

${ }^{8}$ T. Esirkepov, M. Borghesi, S. V. Bulanov, G. Mourou, and T. Tajima, Phys. Rev. Lett. 92, 175003 (2004).

${ }^{9}$ X. Q. Yan, C. Lin, Z. M. Sheng, Z. Y. Guo, B. C. Liu, Y. R. Lu, J. X. Fang, and J. E. Chen, Phys. Rev. Lett. 100, 135003 (2008).

${ }^{10}$ B. Qiao, M. Zepf, M. Borghesi, and M. Geissler, Phys. Rev. Lett. 102, 145002 (2009).

${ }^{11}$ S. Kneip, S. R. Nagel, S. F. Martins, S. P. D. Mangles, C. Bellei, O. Chekhlov, R. J. Clarke, N. Delerue, E. J. Divall, G. Doucas, K. Ertel, F. Fiuza, R. Fonseca, P. Foster, S. J. Hawkes, C. J. Hooker, K. Krushelnick, W. B. Mori, C. A. J. Palmer, K. Ta Phuoc, P. P. Rajeev, J. Schreiber, M. J. V. Streeter, D. Urner, J. Vieira, L. O. Silva, and Z. Najmudin, Phys. Rev. Lett. 103, 035002 (2009).

${ }^{12}$ S. C. Wilks, A. B. Langdon, T. E. Cowan, M. Roth, M. Singh, S. Hatchett, M. H. Key, D. Pennington, A. MacKinnon, and R. A. Snavely, Phys. Plasmas 8, 542-549 (2001). 
${ }^{13}$ H. Schwoerer, S. Pfotenhauer, O. Jackel, K. U. Amthor, B. Liesfeld, W. Ziegler, R. Sauerbrey, K. W. D. Ledingham, and T. Esirkepov, Nature 439, 445-448 (2006).

${ }^{14}$ A. Macchi, S. Veghini, T. V. Liseykina, and F. Pegoraro, New J. Phys. 12, 045013 (2010).

${ }^{15}$ A. Henig, S. Steinke, M. Schnürer, T. Sokollik, R. Hörlein, D. Kiefer, D. Jung, J. Schreiber, B. M. Hegelich, X. Q. Yan, J. Meyer-ter-Vehn, T. Tajima, P. V. Nickles, W. Sandner, and D. Habs, Phys. Rev. Lett. 103, 245003 (2009)

${ }^{16}$ A. Macchi, F. Cattani, T. V. Liseykina, and F. Cornolti, Phys. Rev. Lett. 94, 165003 (2005).

${ }^{17}$ Q. Liu, M. Liu, T. Yu, P. Ding, Z. Liu, S. Sun, X. Liu, X. Lu, Z. Guo, and B. Hu, Phys. Plasmas 19, 093108 (2012).

${ }^{18}$ S. S. Bulanov, A. Brantov, V. Y. Bychenkov, V. Chvykov, G. Kalinchenko, T. Matsuoka, P. Rousseau, S. Reed, V. Yanovsky, D. W. Litzenberg, K. Krushelnick, and A. Maksimchuk, Phys. Rev. E 78, 026412 (2008).

${ }^{19}$ E. Fourkal, I. Velchev, and C. M. Ma, Phys. Rev. E 71, 036412 (2005).

${ }^{20}$ T. Tajima, K. Nakajima, and G. Mourou, Riv. Nuovo Cimento 40, 33 (2017).

${ }^{21}$ F. Pegoraro and S. V. Bulanov, Phys. Rev. Lett. 99, 065002 (2007).

${ }^{22}$ E. M. Weibel, Phys. Rev. Lett. 2, 83 (1959).

${ }^{23}$ G. Kalman, C. Montes, and D. Quemada, Phys. Fluids 11, 1797 (1968).

${ }^{24}$ T. C. Liu, X. Shao, C. S. Liu, M. He, B. Eliasson, V. TripathI, J. J. Su, J. Wang, and S. H. Chen, New J. Phys. 15, 025026 (2013).

${ }^{25}$ H. B. Zhuo, Z. L. Chen, W. Yu, Z. M. Sheng, M. Y. Yu, Z. Jin, and R. Kodama, Phys. Rev. Lett. 105, 065003 (2010).

${ }^{26}$ T. P. Yu, A. Pukhov, G. Shvets, M. Chen, T. H. Ratliff, S. A. Yi, and V. Khudik, Phys. Plasmas 18, 043110 (2011)
${ }^{27}$ T. P. Yu, A. Pukhov, G. Shvets, and M. Chen, Phys. Rev. Lett. 105, 065002 (2010)

${ }^{28}$ B. Shen, X. Zhang, Z. Sheng, M. Y. Yu, and J. Cary, Phys. Rev. Spec. Top. Accel. Beams 12, 121301 (2009).

${ }^{29}$ L. L. Yu, H. Xu, W. M. Wang, Z. M. Sheng, B. Shen, W. Yu, and J. Zhang, New J. Phys. 12, 045021 (2010).

${ }^{30}$ X. Zhang, B. Shen, L. Ji, F. Wang, and M. Wen, Phys. Plasmas 17, $123102(2010)$

${ }^{31}$ F. L. Zheng, S. Z. Wu, H. C. Wu, C. T. Zhou, H. B. Cai, M. Y. Yu, T. Tajima, X. Q. Yan, and X. T. He, Phys. Plasmas 20, 013107 (2013).

${ }^{32}$ W. Yao, B. Li, L. Cao, F. Zheng, T. Huang, C. Xiao, and M. M. Skoric, Laser Part. Beams 32(4), 583-589 (2014).

${ }^{33}$ B. Rau and T. Tajima, Phys. Plasmas 5, 3575 (1998).

${ }^{34}$ M. A. Bake, S. Zhang, B. S. Xie, X. R. Hong, and H. Y. Wang, Phys. Plasmas 19, 083103 (2012).

${ }^{35}$ X. Wang, B. Shen, X. Zhang, W. Wang, J. Xu, L. Yi, and Y. Shi, Phys. Plasmas 22, 043106 (2015).

${ }^{36}$ X. Zhang, B. Shen, L. Ji, W. Wang, J. Xu, Y. Yu, L. Yi, X. Wang, N. A. Hafz, and V. Kulagin, Phys. Plasmas 19, 053103 (2012).

${ }^{37}$ H. Vosoughian, Z. Riazi, H. Afarideh, and G. Sarri, Phys. Plasmas 23, 123113 (2016)

${ }^{38}$ H. Vosoughian, Z. Riazi, H. Afarideh, and G. Sarri, Chin. Phys. B 26, 025201 (2017)

${ }^{39}$ See http://ccpforge.cse.rl.ac.uk/gf/project/epoch/ for EPOCH: Extendable PIC Open Collaboration, 2011.

${ }^{40}$ M. Wen, B. Shen, X. Zhang, F. Wang, Z. Jin, L. Ji, W. Wang, J. Xu, and K. Nakajima, New J. Phys. 12, 045010 (2010). 